\title{
GeoTextMESS: Result Fusion with Fuzzy Borda Ranking in Geographical Information Retrieval
}

\author{
Davide Buscaldi ${ }^{1}$, José M. Perea Ortega ${ }^{2}$, Paolo Rosso ${ }^{1}$, L. Alfonso Ureña \\ López $^{2}$, Daniel Ferrés ${ }^{3}$, and Horacio Rodríguez ${ }^{3}$ \\ 1 Natural Language Engineering Lab, \\ ELiRF Research Group, \\ Dpto. de Sistemas Informáticos y Computación, \\ Universidad Politécnica de Valencia \\ \{dbuscaldi, prosso\}@dsic.upv.es \\ 2 SINAI Research Group, \\ Computer Science Department, \\ Universidad de Jaén \\ \{jmperea, laurena\}@ujaen.es \\ 3 TALP Research Center, \\ Universitat Politècnica de Catalunya \\ \{dferres, horacio\}@lsi.upc.edu
}

\begin{abstract}
In this paper we discuss the integration of different GIR systems by means of a fuzzy Borda method for result fusion. Two of the systems, the one by the Universidad Politécnica de Valencia and the one of the Universidad of Jaén participated to the GeoCLEF task under the name TextMess. The proposed result fusion method takes as input the document lists returned by the different systems and returns a document list where the documents are ranked according to the fuzzy Borda voting scheme. The obtained results show that the fusion method allows to improve the results of the component systems, although the fusion is not optimal, because it is effective only if the components return a similar set of relevant documents.
\end{abstract}

\section{Introduction}

Result fusion has been studied as an option for obtaining better results in Information Retrieval (IR) by taking advantage from the combination of existing methods. Many fusion method have been proposed, such as linear combinations $[1,2]$ and voting schemes like the Condorcet [3] and the Borda [4] schemes. Aslam and Montague [4] concluded that the Borda fusion is a simple, unsupervised method that is capable to exceed the performance of the best component system. The fuzzy Borda voting scheme is an improvement of the standard Borda voting scheme that was introduced by $[5,6]$. This is the first time it is used in the IR task, although it has been used in the Word Sense Disambiguation task at Semeval ${ }^{4}$ with good results [7].

\footnotetext{
${ }^{4}$ http://nlp.cs.swarthmore.edu/semeval
} 
In Sections 2, 3 and 4 we describe briefly the systems of each group. In Section 5 we describe the fuzzy Borda ranking method, in Section 6 we present the experiments carried out and the obtained results. Finally, in Section 7 we draw some conclusions.

\section{The SINAI-GIR System}

The SINAI-GIR system is composed of the following subsystems: the Collection Preprocessing subsystem, the Query Analyzer, the Information Retrieval subsystem and the Validator. Each query is preprocessed and analyzed by the Query Analyzer, identifying its geo-entities and spatial relations and making use of the Geonames gazetteer ${ }^{5}$. This module also applies query reformulation based on the query parsing sub-task, generating several independent queries which will be indexed and searched by means of the IR subsystem. On the other hand, the collection is pre-processed by the Collection Preprocessing module and finally the documents retrieved by the IR subsystem are filtered and re-ranked by means of the Validator subsystem.

The features of each subsystem are:

- Collection Preprocessing Subsystem. During the collection preprocessing, two indexes are generated (locations and keywords indexes). We apply the Porter stemmer, the Brill POS tagger and a the LingPipe ${ }^{6}$ Named Entity Recognizer (NER). We also discard the English stop-words.

- Query Analyzer. It is responsible for the preprocessing of English queries as well as the generation of different query reformulations.

- Information Retrieval Subsystem. As IR index-search engine we have used Lemur ${ }^{7}$.

- Validator. The aim of this subsystem is to filter the lists of documents recovered by the IR subsystem, establishing which of them are valid, depending on the locations and the geo-relations detected in the query. Another important function is to establish the final ranking of documents, based on manual rules and predefined weights.

\section{The NLEL GeoWorSE System}

The system is built around the Lucene ${ }^{8}$ open source search engine, version 2.1. The Stanford NER system based on Conditional Random Fields [8] is used for Named Entity Recognition and classification. The toponym disambiguator is based on the method presented in [9].

During the indexing phase, the documents are examined in order to find location names (toponyms) by means of the Stanford NER system. When a

\footnotetext{
${ }^{5}$ http://www.geonames.org

${ }^{6}$ http://alias-i.com/lingpipe

7 http://www.lemurproject.org

${ }^{8}$ http://lucene.apache.org/
} 
toponym is found, the disambiguator determines the correct reference for the toponym. Then, the toponym coordinates are added to the geo index, and the toponym is stored in the wn index together with its holonyms and synonyms. All document terms are stored in the text index.

The search phase starts with the search of the topic keywords in the text index. The toponyms extracted by the Stanford NER are searched for in the $w n$ index with a weight 0.25 with respect to the content terms. The result of the search is a list of documents ranked using the Lucene's weighting scheme. At the same time, the toponyms are used to define a geographical constraint that is used to re-rank the document list. There are two types of geographical constraints:

- a distance constraint, corresponding to a point in the map: documents that contain locations closer to this point will be ranked higher;

- an area constraint, corresponding to a polygon in the map: documents that contain locations included in the polygon will be ranked higher.

Finally, the documents retrieved by Lucene are re-ranked depending on the geographical constraints.

\section{The TALP GeoIR system}

The TALPGeoIR system [10] has five phases performed sequentially: collection processing and indexing, linguistic and geographical analysis of the topics, textual IR with Terrier, Geographical Retrieval with Geographical Knowledge Bases (GKBs), and geographical document re-ranking.

The collection is processed and indexed in two different indexes: a geographical index with geographical information extracted from the documents and enriched with the aid of GKBs and a textual index with the lemmatized content of the documents.

The linguistic analysis uses the following Natural Language Processing tools: TnT, a statistical POS tagger, the WordNet lemmatizer 2.0, and a Maximum Entropy-based NERC system, trained with the CONLL-2003 shared task English data set.

The retrieval system is a textual IR system based on Terrier [11]. Terrier configuration includes a TF-IDF schema, lemmatized query topics, Porter Stemmer, and Relevance Feedback using 10 top documents and 40 top terms.

The Geographical Retrieval uses geographical terms and/or geographical feature types appearing in the topics to retrieve documents from the geographical index. The geographical search allows to retrieve documents with geographical terms that are included in the sub-ontological path of the query terms (e.g. documents containing Alaska are retrieved from a query United States).

Finally, a geographical re-ranking is performed using the set of documents retrieved by Terrier. From this set of documents those that have been also retrieved in the Geographical Retrieval set are re-ranked giving them more weight than the other ones. 


\section{Fuzzy Borda Fusion}

\subsection{Fuzzy Borda count}

In the classical (discrete) Borda count, each expert gives a mark to each alternative. The mark is given by the number of alternatives worse than it. The fuzzy variant $[5,6]$ allows the experts to show numerically how much alternatives are preferred over others, expressing their preference intensities from 0 to 1 .

Let $R^{1}, R^{2}, \ldots, R^{m}$ be the fuzzy preference relations of $m$ experts over $n$ alternatives $x_{1}, x_{2}, \ldots, x_{n}$. Each expert $k$ expresses its preferences by means of a matrix of preference intensities:

$$
\left(\begin{array}{cccc}
r_{11}^{k} & r_{12}^{k} & \ldots & r_{1 n}^{k} \\
r_{21}^{k} & r_{22}^{k} & \ldots & r_{2 n}^{k} \\
\ldots & \ldots & \ldots & \ldots \\
r_{n 1}^{k} & r_{n 2}^{k} & \ldots & r_{n n}^{k}
\end{array}\right)
$$

where each $r_{i j}^{k}=\mu_{R^{k}}\left(x_{i}, x_{j}\right)$, with $\mu_{R^{k}}: X \times X \rightarrow[0,1]$ is the membership function of $R^{k}$. The number $r_{i j}^{k} \in[0,1]$ is considered as the degree of confidence with which the expert $k$ prefers $x_{i}$ over $x_{j}$. The final value assigned by the expert $k$ to each alternative $x_{i}$ is the sum by row of the entries greater than 0.5 in the preference matrix, or, formally:

$$
r_{k}\left(x_{i}\right)=\sum_{j=1, r_{i j}^{k}>0.5}^{n} r_{i j}^{k}
$$

The threshold 0.5 ensure the relation $R^{k}$ to be an ordinary preference relation.

The fuzzy Borda count for an alternative $x_{i}$ is obtained as the sum of the values assigned by each expert to that alternative: $\mathbf{r}\left(x_{i}\right)=\sum_{k=1}^{m} r_{k}\left(x_{i}\right)$.

\subsection{Application of Fuzzy Borda count to Result Merging}

In our approach each system is an expert: therefore, for $m$ systems, there are $m$ preference matrices. The size of these matrices is variable: the reason is that the document list is not the same for all the systems. The size of a preference matrix is $N_{t} \times N_{t}$, where $N_{t}$ is the number of unique documents retrieved by the systems (i.e. the number of documents that appear at least in one of the lists returned by the systems) for topic $t$.

Each system may rank the documents using weights that are not in the same range of the other ones. Therefore, the output weights $w_{1}, w_{2}, \ldots, w_{n}$ of each expert $k$ are transformed to fuzzy confidence values by means of the following transformation:

$$
r_{i j}^{k}=\frac{w_{i}}{w_{i}+w_{j}}
$$

This transformation ensures that the preference values are in the range $[0,1]$. In order to adapt the fuzzy Borda count to the merging of the results of IR 
systems, we had to determine the preference values in all the cases where one of the systems does not retrieve a document that has been retrieved by another one. The preference values of these documents were set to 0.5 , corresponding to the idea that the expert is presented with an option on which it cannot express a preference.

\section{Experiments and Results}

In Tables 1 and 2 we show the detail of each run in terms of the component systems and the topic fields used. "Official" runs (i.e., the ones submitted to GeoCLEF) are labeled with TMESS02-08 and TMESS07A.

Table 1. Description of the runs of each system.

\begin{tabular}{|c|c|}
\hline run ID & description \\
\hline \multicolumn{2}{|r|}{ NLEL } \\
\hline NLEL0802 & base system (only text index, no wordnet, no map filtering) \\
\hline NLEL0803 & 2007 system (no map filtering) \\
\hline NLEL0804 & base system, title and description only \\
\hline NLEL0505 & 2008 system, all indices and map filtering enabled \\
\hline NLEL01 & complete 2008 system, title and description \\
\hline \multicolumn{2}{|r|}{ SINAI } \\
\hline EXP1 & base system, title and description only \\
\hline EXP2 & base system, all fields \\
\hline EXP4 & filtering system, title and description only \\
\hline EXP5 & filtering system (rule-based) \\
\hline \multicolumn{2}{|r|}{ TALP } \\
\hline TALP01 & system without GeoKB, title and description only \\
\hline TALP02 & complete system, including GeoKB, title and description \\
\hline
\end{tabular}

In order to evaluate the contribution of each system to the final result, we calculated the overlap rate $O$ of the documents retrieved by the systems: $O=$ $\frac{\left|D_{1} \cap \ldots \cap D_{m}\right|}{\left|D_{1} \cup \ldots \cup D_{m}\right|}$, where $m$ is the number of systems that have been combined together and $D_{i}, 0<i \leq m$ is the set of documents retrieved by the $i$-th system. The obtained value measures how different are the sets of documents retrieved by each system.

The $R$-overlap and $N$-overlap coefficients introduced by [12] are used to calculate the degree of overlap of relevant and non-relevant documents in the results of different systems. $R$-overlap is defined as $R_{\text {overlap }}=\frac{m \cdot\left|R_{1}\right| \cdot \ldots \cdot\left|R_{m}\right|}{\left|R_{1}\right|+\ldots+\left|R_{m}\right|}$, where $R_{i}, 0<i \leq m$ is the set of relevant documents retrieved by the system $i . N$ overlap is calculated in the same way, where each $R_{i}$ has been substituted by $N_{i}$, the set of the non-relevant documents retrieved by the system $i$.

In Table 3 we show the Mean Average Precision (MAP) obtained for each run and its composing runs, together with the average MAP calculated over the composing runs. 
Table 2. Details of the composition of all the evaluated runs.

\begin{tabular}{|c|c|c|c|c|}
\hline run ID & fields & NLEL run ID & run ID & TALP run ID \\
\hline \multicolumn{5}{|c|}{ Officially evaluated runs } \\
\hline TMESS02 & TDN & NLEL0802 & EXP2 & \\
\hline TMESS03 & TDN & NLEL0802 & EXP5 & \\
\hline TMESS05 & TDN & NLEL0803 & EXP2 & \\
\hline TMESS06 & TDN & NLEL0803 & EXP5 & \\
\hline TMESS07A & $\mathrm{TD}$ & NLEL0804 & EXP1 & \\
\hline TMESS08 & TDN & NLEL0505 & EXP5 & \\
\hline \multicolumn{5}{|c|}{ Non-official runs } \\
\hline TMESS10 & $\mathrm{TD}$ & & EXP1 & TALP01 \\
\hline TMESS11 & TD & NLEL01 & EXP1 & \\
\hline TMESS12 & $\mathrm{TD}$ & NLEL01 & & TALP01 \\
\hline TMESS13 & TD & NLEL0804 & & TALP01 \\
\hline TMESS14 & $\mathrm{TD}$ & NLEL0804 & EXP1 & TALP01 \\
\hline TMESS15 & TD & NLEL01 & EXP1 & TALP01 \\
\hline
\end{tabular}

Table 3. Results obtained for the various system combinations.

\begin{tabular}{|l|l|r|r|r|r|}
\hline run ID & MAP & $M A P_{N L E L}$ & $M A P_{S I N A I}$ & $M A P_{T A L P}$ & avg. MAP \\
\hline TMESS02 & 0.227 & 0.201 & 0.226 & & 0.213 \\
TMESS03 & 0.219 & 0.201 & 0.212 & & 0.206 \\
TMESS05 & 0.235 & 0.216 & 0.226 & & 0.221 \\
TMESS06 & 0.226 & 0.216 & 0.212 & & 0.214 \\
TMESS07A & 0.286 & 0.256 & 0.284 & & 0.270 \\
TMESS08 & 0.216 & 0.203 & 0.212 & & 0.207 \\
TMESS10 & 0.289 & & 0.284 & 0.280 & 0.282 \\
TMESS11 & 0.285 & 0.254 & & 0.280 & 0.267 \\
TMESS12 & 0.287 & 0.254 & 0.284 & & 0.269 \\
TMESS13 & 0.271 & 0.256 & & 0.280 & 0.268 \\
TMESS14 & 0.282 & 0.256 & 0.284 & 0.280 & 0.273 \\
TMESS15 & 0.289 & 0.254 & 0.284 & 0.280 & 0.273 \\
\hline
\end{tabular}

The obtained results show that the fuzzy Borda merging method always allows to improve the average of the results of the components, and only in two cases it cannot improve the best component result (TMESS13 and TMESS14). The results in Table 4 show that the best results are obtained if the systems returns a similar set of relevant documents (TMESS10 and TMESS12). In order to better understand this result, we calculated the results that would have been obtained by calculating the fusion over different configurations of each group's system. These results are shown in Table 5 .

The fuzzy Borda method allowed also in the case of the fusion of two configurations of the same system to improve the results of the component runs. $O, R_{\text {overlap }}$ and $N_{\text {overlap }}$ values for same-group fusions are well above the $O$ values obtained in the case of different systems (more than 0.73 with respect to $0.31-0.47)$. However, the obtained results show that the method is not able to 
Table 4. $O, R_{\text {overlap }}, N_{\text {overlap }}$ coefficients, difference from the best system (diff. best) and difference from the average of the systems (diff. avg.) for all runs.

\begin{tabular}{|l|r|r|r|r|r|r|}
\hline run ID & MAP & diff. best & diff. avg. & $O$ & $R_{\text {overlap }}$ & $N_{\text {overlap }}$ \\
\hline TMESS01 & 0.226 & 0.001 & 0.013 & 0.315 & 0.698 & 0.459 \\
TMESS02 & 0.227 & 0.001 & 0.014 & 0.346 & 0.692 & 0.496 \\
TMESS03 & 0.219 & 0.007 & 0.013 & 0.317 & 0.693 & 0.465 \\
TMESS05 & 0.235 & 0.009 & 0.014 & 0.358 & 0.692 & 0.508 \\
TMESS06 & 0.226 & $\mathbf{0 . 0 1 0}$ & 0.012 & 0.334 & 0.693 & 0.484 \\
TMESS07A & 0.286 & 0.002 & 0.016 & 0.356 & 0.775 & 0.563 \\
TMESS08 & 0.216 & 0.004 & 0.013 & 0.326 & 0.690 & 0.475 \\
TMESS10 & $\mathbf{0 . 2 8 9}$ & 0.005 & 0.007 & 0.485 & $\mathbf{0 . 8 5 4}$ & 0.625 \\
TMESS11 & 0.285 & 0.005 & $\mathbf{0 . 0 1 8}$ & 0.475 & 0.796 & 0.626 \\
TMESS12 & 0.287 & 0.003 & $\mathbf{0 . 0 1 8}$ & 0.356 & 0.822 & $\mathbf{0 . 3 5 6}$ \\
TMESS13 & 0.271 & -0.009 & 0.003 & 0.475 & 0.796 & 0.626 \\
TMESS14 & 0.282 & -0.002 & 0.009 & 0.284 & 0.751 & 0.429 \\
TMESS15 & $\mathbf{0 . 2 8 9}$ & 0.005 & 0.016 & 0.277 & 0.790 & 0.429 \\
\hline
\end{tabular}

Table 5. Results obtained with the fusion of systems from the same participant. $M_{1}$ : MAP of the system in the first configuration, $M_{2}$ : MAP of the system in the second configuration.

\begin{tabular}{|l|r|r|r|r|r|r|}
\hline run ID & MAP & $M_{1}$ & $M_{2}$ & $O$ & $R_{\text {overlap }}$ & $N_{\text {overlap }}$ \\
\hline EXP1+EXP4 & 0.289 & 0.284 & 0.275 & 0.792 & 0.904 & 0.852 \\
NLEL0804+NLEL01 & 0.261 & 0.254 & 0.256 & 0.736 & 0.850 & 0.828 \\
TALP01+TALP02 & 0.283 & 0.280 & 0.272 & 0.792 & 0.904 & 0.852 \\
\hline
\end{tabular}

combine in an optimal way the systems that returns different sets of relevant documents. This is due to the fact that a relevant document that is retrieved by a system and not by another one has a 0.5 weight in the preference matrix, making that its ranking will be worse than a non-relevant document retrieved by both systems.

\section{Conclusions and Further Work}

We combined different systems by means of the fuzzy Borda voting scheme. The implemented method allowed to improve in most cases the results of the combined systems, although the improvement was limited. The best results with this method were obtained when the systems returned a similar set of relevant documents, which means that the method needs to be improved in order to better combine sets of different relevant results. This could be done by assigning to the unknown documents a weight different from 0.5 , calculating the similarity of these documents with the ones that have been retrieved by the system. This will be the focus of future research efforts. 


\section{Acknowledgements}

We would like to thank the TextMESS TIN2006-15265-C06 research project and its subprojects TIN2006-15265-C06-04 MiDeS, TIN2006-15265-C06-04-05 and TIN200615265-C06-03 TIMOM for partially supporting this work. Daniel Ferrés is supported by a UPC-Recerca grant.

\section{References}

1. Bartell, B.T., Cottrell, G.W., Belew, R.K.: Automatic combination of multiple ranked retrieval systems. In: SIGIR '94: Proceedings of the 17th annual international ACM SIGIR conference on Research and development in information retrieval, New York, NY, USA, Springer-Verlag New York, Inc. (1994) pp. 173-181

2. Vogt, C.C., Cottrell, G.W.: Fusion via a linear combination of scores. Information Retrieval 1(3) (1999) pp. 151-173

3. Montague, M., Aslam, J.A.: Condorcet fusion for improved retrieval. In: CIKM '02: Proceedings of the eleventh international conference on Information and knowledge management, New York, NY, USA, ACM (2002) pp. 538-548

4. Aslam, J.A., Montague, M.: Models for metasearch. In: SIGIR '01: Proceedings of the 24th annual international ACM SIGIR conference on Research and development in information retrieval, New York, NY, USA, ACM (2001) pp. 276-284

5. Nurmi, H.: Resolving Group Choice Paradoxes Using Probabilistic and Fuzzy Concepts. Group Decision and Negotiation 10(2) (2001) pp. 177-199

6. García Lapresta, J., Martínez Panero, M.: Borda Count Versus Approval Voting: A Fuzzy Approach. Public Choice 112(1-2) (2002) pp. 167-184

7. Buscaldi, D., Rosso, P.: Upv-wsd : Combining different wsd methods by means of fuzzy borda voting. In: Fourth International Workshop on Semantic Evaluations (SemEval-2007), ACL (2007) pp. 434-437

8. Finkel, J.R., Grenager, T., Manning, C.: Incorporating non-local information into information extraction systems by gibbs sampling. In: Proceedings of the 43nd Annual Meeting of the Association for Computational Linguistics (ACL 2005), U. of Michigan - Ann Arbor, ACL (2005) pp. 363-370

9. Buscaldi, D., Rosso, P.: A conceptual density-based approach for the disambiguation of toponyms. International Journal of Geographical Information Systems 22(3) (2008) pp. 301-313

10. Ferrés, D., Rodríguez, H.: TALP at GeoCLEF 2007: Results of a Geographical Knowledge Filtering Approach with Terrier. In: Advances in Multilingual and Multimodal Information Retrieval, 8th Workshop of the Cross-Language Evaluation Forum, CLEF 2007, Budapest, Hungary, September 19-21, 2007, Revised Selected Papers. Springer, Budapest, Hungary (2008) pp. 830-833

11. Ounis, I., Amati, G., Plachouras, V., He, B., Macdonald, C., Lioma, C.: Terrier: A High Performance and Scalable Information Retrieval Platform. In: Proceedings of ACM SIGIR'06 Workshop on Open Source Information Retrieval (OSIR 2006). (2006)

12. Lee, J.H.: Analyses of multiple evidence combination. In: SIGIR '97: Proceedings of the 20th annual international ACM SIGIR conference on Research and development in information retrieval, New York, NY, USA, ACM (1997) pp. 267-276 